\title{
Modelling Coarseness in Texture Images by Means of Fuzzy Sets
}

\author{
J. Chamorro-Martínez, E. Galán-Perales, D. Sánchez, and J.M. Soto-Hidalgo \\ Department of Computer Science and Artificial Intelligence \\ University of Granada \\ C/ Periodista Daniel Saucedo Aranda s/n, 18071 Granada, Spain \\ \{jesus, elena, daniel, soto\}@decsai.ugr.es
}

\begin{abstract}
In this paper we model the concept of "coarseness", typically used in texture image descriptions, by means of fuzzy sets. Specifically, we relate representative measures of this kind of texture with its presence degree. To obtain these "presence degrees", we collect assessments from polls filled by human subjects, performing an aggregation of these assessments by means of OWA operators. Using this collected data, and some statistics as reference set, the membership function corresponding to the fuzzy set "coarseness" is modelled.
\end{abstract}

Keywords: Image features, texture features, fuzzy texture, visual coarseness.

\section{Introduction}

Visual texture is one of the most difficult features to be characterized in images due to the imprecission of the concept itself. In fact, there is not an accurate definition for the concept of texture but some widespread intuitive ideas. In this way, texture is described by some authors as local changes in the intensity patterns or gray tones. Other authors consider texture as a set of basic items called texels (or texture primitives), arranged in a certain way [12. Moreover, it is usual for humans to describe visual textures according to some "textural concepts" like coarseness, orientation, regularity [3]. To describe such concepts, linguistic labels are used (e.g. coarse or fine can be used to describe coarseness).

The own imprecision of the concept of texture suggests to use representation models that incorporate the uncertainty. Nevertheless, the majority of the approaches found in the literature are crisp proposals 4 where uncertainty is not properly taken into account. To deal with the imprecission relative to visual texture, there are some approaches which introduce the use of fuzzy logic [5]. However, in many of them, fuzzy logic is often applied just during the process but the output do not usually model the imprecission (being often a crisp one).

In this paper we focus our study on coarseness, one of the textural properties most used in the literature which allows to distinguish between fine and coarse

\footnotetext{
* This work has been partially supported by "Instituto de Salud Carlos III under FIS
} G03/185 project, "Imagen Médica Molecular y Multimodalidad".

B. Gabrys, R.J. Howlett, and L.C. Jain (Eds.): KES 2006, Part II, LNAI 4252, pp. 355-362 2006. (C) Springer-Verlag Berlin Heidelberg 2006 

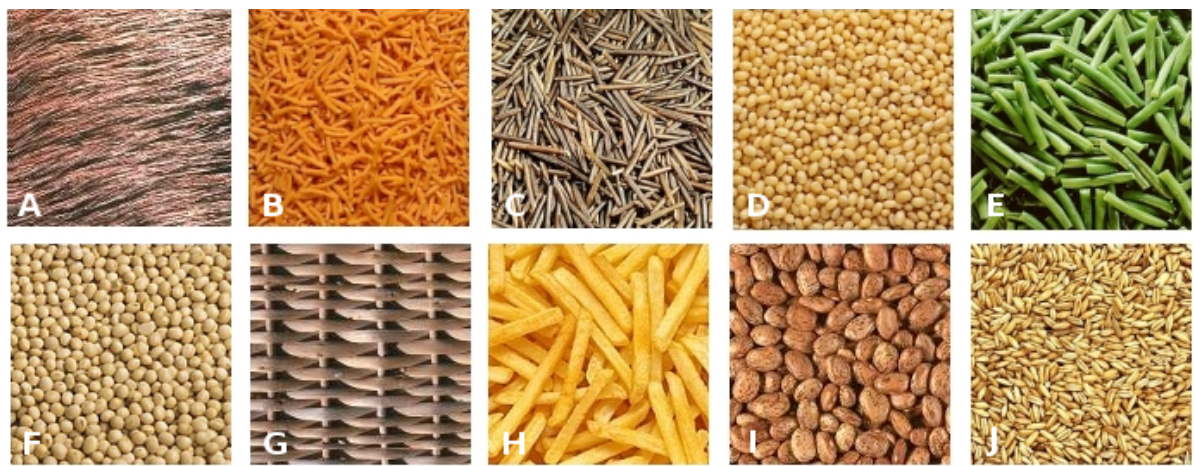

Fig. 1. Some examples of images with different degrees of fineness

textures. In fact, It is usual that the texture concept is associated to the presence of fineness. A fine texture can be considered as small texture primitives with big gray tone differences between neighbour primitives (e.g. the image in figure 1(A)). On the contrary, if texture primitives are bigger and formed by several pixels, it is a coarse texture (e.g. the image in figure 1(A)).

In this paper, we propose to model fineness by means of fuzzy sets to deal with the problem of imprecision found in texture characterization. To do this, two questions will be faced: what reference set should be used for the fuzzy set, and how to obtain the related membership functions. To solve the first question, a vector of measures will be automatically computed from the texture image. To answer the second question, functional relationship between a certain measure and the presence degree of a textural concept related to it will be learnt.

The rest of the paper is organized as follows. In section 2 we introduce our methodology to obtain the fuzzy sets related to fineness textural concept. In section 3 we show the results of applying the model and the main conclusions and future work are sumarized in section 4 .

\section{Texture Modelling. Application to Fineness}

In this paper, we propose to model a textural concept as a fuzzy set. From now on, we shall denote $\mathcal{T}$ the textural concept we want to model, in our case $\mathcal{T}=$ fineness. As reference set, a vector of $K$ measures obtained by carrying out an analysis of the texture image is used. These measures should give reliable information about the presence degree of the textural concept fineness under study. Thus, the model of the textural concept will be given by a fuzzy subset built on the domain of the chosen measure.

Furthermore, a membership function that models the textural concept fineness for the fuzzy set is needed. In this paper we propose to obtain this function by learning a functional relationship between a certain measure and the presence degree of the textural concept fineness. 
To learn this relationship, we will use a set $\mathcal{I}=\left\{I_{1}, \ldots, I_{N}\right\}$ of $N$ images that fully represent the textural concept $\mathcal{T}$ to be learnt. Given the concept $\mathcal{T}$, a set of measures $\mathcal{P}=\left\{P_{1}, \ldots, P_{K}\right\}$ will be considered, with $P_{k} \in \mathcal{P}$ being a measure of the presence of $\mathcal{T}$ in an image (e.g. in the case of $\mathcal{T}=$ fineness we could define $\mathcal{P}=\{$ EdgeDensity, Variance, Range $\}$ ). Thus, for each image $I_{i} \in \mathcal{I}$, we will obtain (a) a vector of measures $\mathbf{M}^{i}=\left[m_{1}^{i}, \ldots, m_{K}^{i}\right]$, where $m_{k}^{i}$ is a value for the measure $P_{k} \in \mathcal{P}$ applied to the image $I_{i}$ (section 2.1), and (b) an assessment $v^{i}$ of the presence degree of the textural concept $\mathcal{T}$ under study. To get this assessment, a poll will be performed (section 2.2). Once we have a multiset of valid pairs $\Psi=\left\{\left(\mathbf{M}^{1}, v^{1}\right), \ldots,\left(\mathbf{M}^{N}, v^{N}\right)\right\}$, we shall estimate the membership function (section 2.4).

\subsection{Fineness Measures}

Given the textural concept $\mathcal{T}=$ fineness a set of measures $\mathcal{P}=\left\{P_{1}, \ldots, P_{K}\right\}$ will be considered. Different measures that characterize the presence of fine texture are found over the literature [3. In this paper we have chosen simple measures which imply a low computational cost. We have specifically used three well known measures:

- Range, measured as the difference between the minimum and the maximum values in the image. We will note Range $e^{i}$ the range related to the image $I_{i}$

- Variance of the image gray tones. We will note $\operatorname{Var}^{i}$ the variance related to the image $I_{i}$

- Edge density, measured as the percentage of points which are an edge in the image. In this paper, we have used the Canny Edge Detector [6]. We will note EdgeDens ${ }^{i}$ the edge density related to the image $I_{i}$

In section 2.3 we will study the goodness of a measure $P_{k} \in \mathcal{P}$ to model a textural concept $\mathcal{T}$. This study will be performed by analyzing the ability of $P_{k}$ to discriminate the assessments given by a set of subjects.

\subsection{Assessment Collection}

In this section we will describe how to get, from the image set $\mathcal{I}=\left\{I_{1}, \ldots, I_{N}\right\}$, a vector $\Gamma=\left[v^{1}, \ldots, v^{N}\right]$ of the assessments of the presence degrees related to the textural concept $\mathcal{T}=$ fineness.

Thus, firstly a criterion for choosing the image set $\mathcal{I}$ is needed. After that, a poll which allows to get assessments of the presence degree of the textural concept $\mathcal{T}$ will be designed. These assessments will be obtained for each image in $\mathcal{I}$. Finally, to get just one assessment that summarizes the information given by human subjects, an aggregation of the different assessments will be performed.

The texture image set. Firstly, the image set $\mathcal{I}=\left\{I_{1}, \ldots, I_{N}\right\}$ that fully represents the textural concept to be learnt must be chosen. In this paper, we have selected a set $\mathcal{I}$ of $N=80$ images representative of the concept of fineness. Figure 1 shows some images extracted from the set $\mathcal{I}$. The selection was done to 
cover the different presence degrees of fineness with a representative number of images. Furthermore, we have selected images in which, as far as possible, just one degree of fineness is perceived.

The poll. Given the image set $\mathcal{I}$, the next step is to obtain assessments about the perception of $\mathcal{T}$ from a set of subjects. From now on we shall denote $\Theta^{i}=$ $\left[o_{1}^{i}, \ldots, o_{L}^{i}\right]$ the vector of assessments obtained from $L$ subjects for image $I_{i}$. To get $\Theta^{i}$ we will ask subjects to assign images to classes, so that each class has associated a presence degree. The number of classes is fixed and an example image which represents the presence degree is associated to each class.

In particular, 20 subjects have participated in the poll and 9 classes $C_{1}, C_{2}$, $\ldots, C_{9}$ have been considered. The first nine images in figure 1 show the nine representative images for each class $C_{k}, k=1, \ldots, 9$ used in this poll. It should be noticed that the images are decreasingly ordered according to the presence degree of the fineness concept. The first class $C_{1}$ (figure 1(A)), represents a presence degree of 1 while the ninth class $C_{9}$ (figure 1(I)), represents a presence degree of 0 . The rest of the classes (figure $1(B)-(H),(J)$ ) represent degrees in the interval $(0,1)$.

Finally, a vector of 20 assessments $\Theta^{i}=\left[o_{1}^{i}, \ldots, o_{20}^{i}\right]$ is obtained for each image $I_{i} \in \mathcal{I}$. The degree $o_{j}^{i}$ associated to the assessment given by the subject $S_{j}$ to the image $I_{i}$ is computed as $o_{j}^{i}=(9-k) * 0.125$, where $k$ is the index of the class $C_{k}$ to which the image is assigned by the subject $S_{j}$.

Assessment aggregation. Our aim at this point is to obtain, for each image in the set $\mathcal{I}$, one assessment that summarizes the assessments given by the different subjects about the presence degree of fineness.

To aggregate opinions we have chosen the use of OWA operator guided by a quantifier [7. With these operators it can be represented the existing interval between logic $A N D$, which allows for the representation of the quantifier for all, and logic $O R$, which allows for the representation of the quantifier exists.

Yager proposed in [7] the use of monotonically nondecreasing linguistic quantifiers. In particular, we can use quantifiers of the form:

$$
Q(r)=\left\{\begin{array}{lr}
0 & \text { if } r<a, \\
\frac{r-a}{b-a} \text { if } a \leq r \leq b, \\
1 & \text { if } r>b
\end{array}\right.
$$

being $a, b, r \in[0,1]$. Depending on the values associated to the pair $(a, b)$, the quantifier interpretation would be different. Thus, in [8] the quantifier most is associated to the pair $(0.3,0.8)$, the quantifier at least half $(0,0.5)$ and the quantifier as many as possible $(0.5,1)$. In our proposal, the quantifier most is chosen.

Once the quantifier $Q$ has been chosen, the weighting vector of the OWA operator can be obtained following Yager as $w_{j}=Q(j / L)-Q((j-1) / L), j=$ $1,2, \ldots, L$. According to this, given a quantifier $Q$, for each image $I_{i} \in \mathcal{I}$ the vector $\Theta^{i}$ obtained from $L$ subjects will be aggregated into one assessment $v^{i}$ as follows: 


$$
v^{i}=w_{1} \hat{o}_{1}^{i}+w_{2} \hat{o}_{2}^{i}+\ldots+w_{L} \hat{o}_{L}^{i}
$$

where $\left[\hat{o}_{1}^{i}, \ldots, \hat{o}_{L}^{i}\right]$ is a vector obtained by ranking in nonincreasing order the values of the vector $\Theta^{i}$.

\subsection{Goodness of a Measure $P_{k}$}

Given a measure $P_{k} \in P$ we need to solve two questions: (a) is $P_{k}$ a good measure to appropriately represent $\mathcal{T}$ ? and (b) what is the ability of $P_{k}$ to discriminate different presence degrees for $\mathcal{T}$, i.e. how many classes can $P_{k}$ actually discriminate?

To face the first question, in this paper we propose to perform an ANOVA test to find out if $P_{k}$ can discriminate at least two classes with a significance level of 0.05 . In the test, the measure values obtained by applying $P_{k}$ to each $I_{i} \in \mathcal{I}$ will be the dependent variable, and the class to which the image $I_{i}$ has been (mostly) assigned will be the independent variable.

To face the second question, we propose to apply a set of multiple comparison tests to find out how many classes can $P_{k}$ discriminate. Concretely, we will use the algorithm 1 which, starting with an initial partition, will iteratively join clusters until the partition has a number of classes that can be discriminated. In our proposal, the initial partition $\operatorname{Part}^{0}=C_{1}, C_{2}, \ldots, C_{n c}$ will be the 9 classes, where $C_{r} \in \operatorname{Part}^{0}$ contains the images assigned to the class by the majority of the subjects (section 2.2), as $\delta$ we propose to use the Mahalanobis distance by considering the mean and the variance of the involved classes, as $\phi$ the multiple comparison tests: Scheffé, Bonferroni, Duncan, Tukey's least significant difference, Tukey's honestly significant difference will be considered, and finally the number of positive tests to accept distinguishability will be fixed to $N T=3$ between a pair of clusters.

From now on we shall denote $\widetilde{\text { Part }}_{k}=C_{1}, C_{2}, \ldots, C_{\widetilde{n c}}$, as the classes which can be discriminated by $P_{k}$.

In the case of fineness, from the set $\mathcal{P}=\{$ Range, Variance, EdgeDensity $\}$ considered in this paper, only the measure EdgeDensity passes the ANOVA test and the number of classes obtained from the algorithm 1 for this measure is 3 .

\subsection{Obtaining the Membership Function}

In this section we will deal with the problem of obtaining the membership function for the textural concept $\mathcal{T}=$ fineness. To simplify the notation, as it is usual in the scope of fuzzy sets, we will use the same notation for the textural concept, for the fuzzy set which represents it and for the membership function that defines it. In our proposal, only the measure Edge density will be used to obtain the membership function that models fineness as the rest of the measures of $P$ did not pass the ANOVA test. In this way, the fineness will be modelled by a membership function defined on $\mathbb{R}$ i.e.,

$$
\mathcal{T}: \mathbb{R} \rightarrow[0,1]
$$




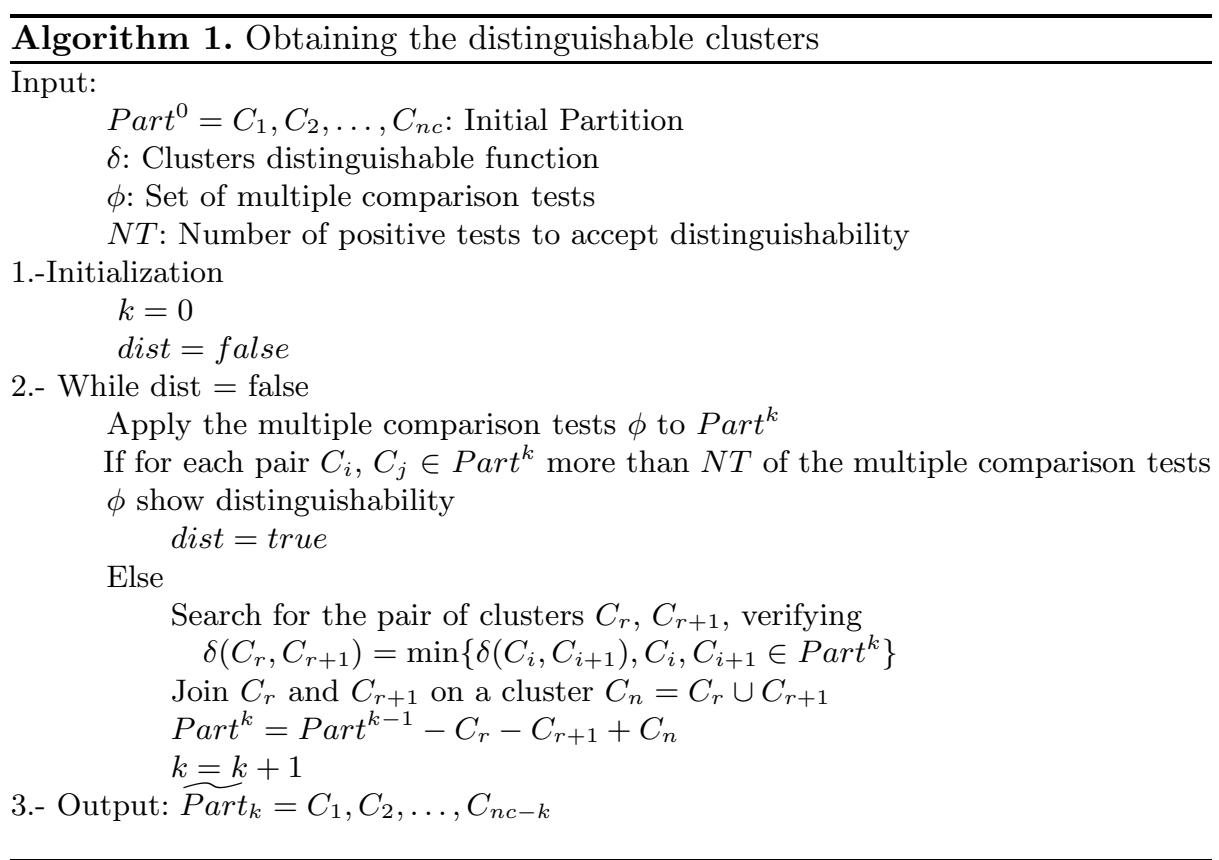

In this paper we propose to define $\mathcal{T}$ as a linear spline, i.e.,

$$
\mathcal{T}(x)=\left\{\begin{array}{lr}
0 & x<=x_{1} \\
T^{1}(x) & x \in\left(x_{1}, x_{2}\right] \\
T^{2}(x) & x \in\left(x_{2}, x_{3}\right] \\
\vdots & \vdots \\
1 & \left.x>x_{\widetilde{n c}}\right]
\end{array}\right.
$$

with $x_{r} r=1 \ldots \widetilde{n c}$, calculated as the mean of the measure values in the class $C_{r} \in \widetilde{\text { Part }}_{k}, T^{r}(x)$ is the straight line defined between the points $\left(x_{r}, y_{r}\right)$ and $\left(x_{r+1}, y_{r+1}\right)$ with $y_{r}$ being the mean value of the presence degree of the textural concept $\mathcal{T}$ for the images grouped into the cluster $C_{r}$.

In our case, as we obtained three different classes, the above menstioned function is defined as follows:

$$
\mathcal{T}(x)=\left\{\begin{array}{lr}
0 & x<=0.17 \\
3.89 * x-0.68 x \in & (0.17,0.30] \\
7.90 * x-1.89 x \in & 0.30,0.37] \\
1 & x>0.37]
\end{array}\right.
$$

\section{Results}

Let's consider figure 2(A) corresponding to a mosaic made by several images, each one with a different increasing fineness presence degree. For each image in 

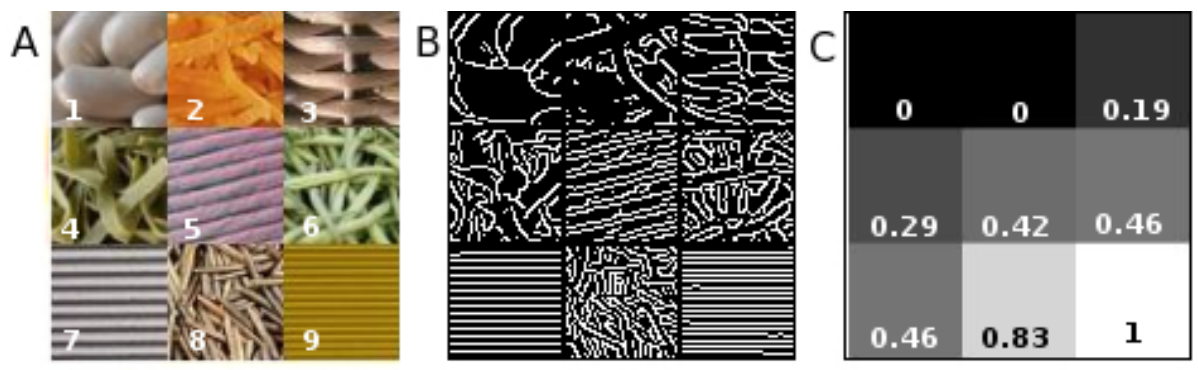

Fig. 2. Results for a mosaic image: (A) original mosaic image (B) edge map of the original images $(\mathrm{C})$ presence degree of fineness textural concept obtained with the proposed model

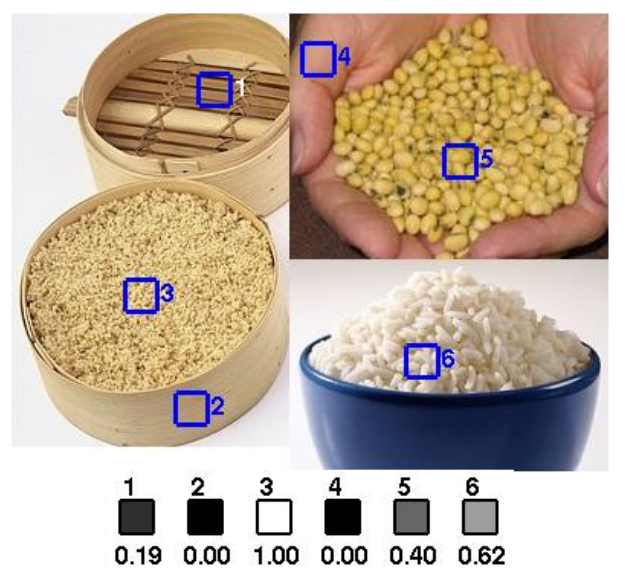

Fig. 3. Some examples of fineness degrees computed for six real subimages

the mosaic, we apply the membership function defined in equation 5 , The image 2(B) shows the edge map obtained from the original mosaic. This map is used to get the edge density which is the reference set of our fuzzy set. The fineness presence degree for each subimage is shown in figure 2 (C) where a white grey level means maximum membership degree, while a black one corresponds to 0 membership degree (the numeric value is also shown on each subimage). It can be noticed that our model captures the evolution of the fineness degrees.

We have also compared our model output with the assessments obtained from subjects. To get them we have aggregated the assessments of 20 subjects following the steps explained in section 2.2. The average error obtained is 0.089 , which shows the goodness of our approach to represent the subjectivity found in fineness perception.

Figure 3 shows several real images where some windows have been selected corresponding to subimages with different fineness degree. We apply the model to each subimage, and the fineness degree obtained is shown at the bottom of 
figure 3. As it can be noticed, our model assesses high degrees to fine texture areas and low degrees to coarse texture areas.

\section{Conclusions and Future Works}

In this paper we have proposed a methodology to represent the fineness concept by means of fuzzy sets. To define the membership function associated to the fuzzy set, the functional relationship between a certain measure (automatically computed over the image) and the presence degree of fineness has been learnt.

In order to obtain the perception degree of a certain textural concept, a group of 20 human subjects have been polled and their assessments have been aggregated by means of OWA operators. After that and by using as reference set a group of very simple statistical measures, we have obtained a fuzzy set which models the human perception of fineness. The results given by our approach show a high level of connection with the assessments given by subjects.

As future work, more complex statistical measures, new ways of defining the membership function and the use of linguistic labels for this fuzzy set in image retrieval by content will be analyzed.

\section{References}

1. Tuceryan, M., Jain, A.: Texture Analysis. In: The Handbook of Pattern Recognition and Computer Vision. 2 edn. C.H. Chen and L.F. Pau and P.S.P. Wang (1998) 207-248

2. Shapiro, L.G., Stockman, G.: Image Segmentation. In: Computer Vision. PrenticeHall (2001) 297-301

3. Abbadeni, N., Ziou, D., Wang, S.: Perceptual textural features corresponding to human visual perception. In: Proc. of the Thirteenth Vision Interface Conference, Montreal, Quebec (Canada) (2000) 365-372

4. Reed, T.R., Buf, J.H.D.: A review of recent texture segmentation and feature extraction techniques. CVGIP: Image Understanding 57(3) (1993) 359-372

5. Shackelford, A.: A hierachical fuzzy classification approach for high-resolution multispectral data over urban areas. IEEE Transactions on Geoscience and Remote Sensing 41(9) (2003) 1920-1932

6. Canny, J.: A computational approach to edge detection. IEEE Transactions on Pattern Analysis and Machine Intelligence 8(6) (1986) 679-698

7. Yager, R.: On ordered weighted averaging aggregation operators in multicriteria decisionmaking. IEEE Transactions on Systems, Man and Cybernetics 18(1) (1988) 183-190

8. Herrera, F., Herrera-Viedma, E.: Linguistic decision analysis: steps for solving decision problems under linguistic information. Fuzzy Sets and Systems 115(1) (2000) $67-82$ 